allergens, to the subject of both indoor and outdoor pollution and to climate change, exposure to environmental tobacco smoke and to methods of heating within the home, to diet and drug use, obesity, and to rates of caesarean sections and breast feeding. ${ }^{10-14}$ Some are hypothesis generating but none has given a comprehensive overarching explanation for a rise in prevalence.

The hypotheses have arisen from a desire to explain epidemiological differences, and those such as the "hygiene" hypothesis had a seemingly corroboratory immunological explanation. ${ }^{15}$ However, they have not taken us to the point where we can proudly announce a primary preventive strategy. This is likely to involve a multipronged approach. Answers are only likely to result from greater study of high risk infants where the study participants are carefully phenotyped, there are few drop outs, tight observation on environmental factors including exposure to infections, and prolonged follow-up. Other studies have identified genetic markers of susceptibility to asthma and been followed by studies that better characterise the clinical risk of such genetic susceptibilities. ${ }^{16}$ The peptic ulcer story may have relevance to asthma and, after early interest in the importance of respiratory syncytial viruses and subsequent excitement at the possible role of Mycoplasma pneumoniae, ${ }^{17}$ we need to recognise that it may be many bacteria that play a part and that some may be protective, some may be aetiologically significant, and that the timing of such infections in the newborn's life may be the critical factor. ${ }^{18}$ Parallel studies are then needed to see whether other genotypic variants inter-react with, for example, air pollution to enhance risk and how this is influenced by exposure to allergens and infections. ${ }^{19}$

We cannot afford to wait another 10 or 20 years for our preventive strategy in asthma. ISAAC has demonstrated the challenge, and large well-funded cohort studies linked to good laboratory documentation of infections, followed hopefully by intervention studies, are needed. In asthma we are far ahead of the diet stage, for we do of course have treatment capable of fully controlling asthma for the majority. However, for the individual and for nations, not developing the disease in the first place would be infinitely preferable. There is a danger that the very effectiveness of treatment reduces the burden to the extent that the funding and impetus to research causation might lessen. That must not be allowed to happen.

Competing interests: None.

Thorax 2009;64:462-463. doi:10.1136/thx.2008.114157

\section{REFERENCES}

1. Asher MI, Keil U, Anderson HR, et al. International Study of Asthma and Allergies in Childhood (ISAAC): rationale and methods. Eur Respir J 1995;8:483-91.

2. Beasley R, Keil U, Von Mutius E, et al. Worldwide variation in prevalence of symptoms of asthma, allergic rhinoconjunctivitis and atopic eczema: ISAAC Lancet 1998;351:1225-32.

3. Pearce N, At-Khaled N, Beasley R, et al. Worldwide trends in the prevalence of asthma symptoms: phase III of the International Study of Asthma and Allergies in Childhood (ISAAC). Thorax 2007;62:758-66.

4. Lai CKW, Beasley R, Crane J, et al. Global variation in the prevalence and severity of asthma symptoms: phase three of the International Study of Asthma and Allergies in Childhood (ISAAC). Thorax 2009;64:476-83.

5. Sears MR, Lewis S, Herbison GP, et al. Comparison of reported prevalences of recent asthma in longitudinal and cross-sectional studies. Eur Respir $J$ 1997:10:51-4

6. Chinn S, Jarvis D, Burney P, et al. Increase in diagnosed asthma but not symptoms in the European Community Respiratory Health Survey. Thorax 2004;59:646-51.

7. Ninan TK, Russell G. Respiratory symptoms and atopy in Aberdeen schoolchildren: evidence from two surveys 25 years apart. BMJ 1992;304:1157.

8. Burr ML, Butland BK, King $\mathrm{S}$, et al. Changes in asthma prevalence: two surveys 15 years apart. Arch Dis Child 1989;64:1452-6.

9. Rona RJ, Chinn S, Burney PG. Trends in the prevalence of asthma in Scottish and English primary school children 1982-92. Thorax 1995;50:992-3.

10. Lewis S, Butland B, Strachan D, et al. Study of aetiology of wheezing illness at age 16 in two national British birth cohorts. Thorax 1996;51:670-6.

11. Roduit C, Scholtens S, de Jongste JC, et al. Asthma at 8 years of age in children born by caesarean section. Thorax 2009;64:107-13.

12. Shaheen So, Sterne JAC, Songhurst CE, et al. Frequent paracetamol use and asthma in adults. Thorax 2000;55:266-70.

13. Oddy WH, Sherriff JL, de Klerk NH, et al. The relation of breastfeeding and body mass index to asthma and atopy in children: a prospective cohort study to age 6 years. Am J Pub Health 2004;94:1531-7.

14. Chinn S, Rona RJ. Can the increase in body mass index explain the rising trend in asthma in children. Thorax 2001;56:845-50.

15. Strachan DP. Hay fever, hygiene, and household size. BMJ 1989;299:1259-60.

16. Bisgaard H, BØnnelykke K, Sleiman PMA, et al. Chromosome 17q21 gene variants are associated with asthma and exacerbations but not atopy in early childhood. Am J Respir Crit Care Med 2009;179:179-85.

17. Yano T, Ichikawa Y, Komatu S, et al. Association of Mycoplasma pneumonia antigen with initial onset of bronchial asthma. Am J Respir Crit Care Med 1994;149:1348-53.

18. Bisgaard $\mathbf{H}$, Hermansen MN, Buchvald F, et al. Childhood asthma after bacterial colonization of the airway in neonates. $N$ Engl $\mathrm{J}$ Med 2007;357:1487-95.

19. Salam MY, Lin P-L, Avol E, et al. Microsomal epoxide hydrolase, glutathione S-transferase P1, traffic and childhood asthma. Thorax 2007;62:1050-7.

\title{
Unintended consequences in the drive for zero
}

\section{Michael Klompas}

With pressure mounting from legislators, insurers and consumer advocates to drive ventilator-associated pneumonia (VAP) rates to zero, the article by Conway Morris and colleagues (see page 516) in this issue of Thorax is a timely reminder

Correspondence to: Dr Michael Klompas, Department of Ambulatory Care and Prevention, Harvard Medical School and Harvard Pilgrim Health Care, 133 Brookline Avenue, 6th Floor, Boston, MA 02215, USA;

mklompas@partners.org that VAP rates are uncertain estimates rather than concrete measures of patient morbidity. ${ }^{1}$ Conway Morris and colleagues show that the reported VAP rate of an Intensive Care Unit (ICU) is heavily dependent upon its favoured diagnostic technique. ICUs that exclusively use bronchoalveolar lavage (BAL) to diagnose $\mathrm{VAP}$ are liable to report VAP rates that are $76 \%$ lower than those that exclusively use endotracheal aspirates. This laxity in the VAP definition confers a risk that some well-intended initiatives may decrease VAP rates yet provide little benefit to patients and perhaps even put some at risk.

Conway Morris and colleagues base their estimate of varying VAP rates upon the comparative yield of simultaneous BAL and endotracheal aspirate cultures taken from 53 critically ill patients with clinical syndromes suggestive of pneumonia. All patients had radiographic infiltrates, pyrexia or an abnormal leucocyte count, and clinical signs of chest infection such as increased volume or purulence of sputum, crepitations and deterioration in oxygenation. In these patients, there was a clear step function in culture results: $89 \%$ of endotracheal aspirate qualitative cultures were positive (defined as any growth), $51 \%$ of endotracheal quantitative 
cultures were positive (defined as $\geqslant 10^{6}$ colony-forming units (CFU)/ml), and $21 \%$ of BAL cultures were positive (defined as $\geqslant 10^{4} \mathrm{CFU} / \mathrm{ml}$ ).

Each of these culture thresholds qualifies as VAP using the definitions of the Hospitals in Europe Link for Infection Control through Surveillance (HELICS) system. Consequently, depending upon one's choice of diagnostic technique, measured VAP rates can vary fourfold. HELICS does assign different VAP codes to patients depending upon which culture technique and threshold was used to establish the case, but these distinctions are seldom if ever cited when hospitals report their VAP rates. For the purposes of most public or government reporting, a VAP is a VAP regardless of diagnostic method.

Conway Morris et al modelled the impact of diagnostic technique on VAP rates and antibiotic usage by applying their data on differential culture yields to their medical-surgical ICU's surveillance and dispensing data from the previous year. They estimated that exclusive use of endotracheal aspirates would have yielded a VAP rate of 25.5 cases per 1000 ventilator days whereas exclusive use of BAL would have yielded 6.1 cases per 1000 ventilator days. They further estimated that exclusive use of BAL would have led to a $30 \%$ reduction in antibiotic use since fewer patients would have had positive cultures.

Conway Morris and colleagues used these findings to catalyse a practice improvement initiative aimed at increasing the use of BAL in their ICU. They managed to increase the rate of bronchoscopy in patients with suspected VAP from $37 \%$ in the year prior to the intervention to $58 \%$ in the year following intervention. As predicted, their observed incidence of VAP dropped by $50 \%$ $(p=0.001)$ while antibiotic usage declined by $21 \%(p=0.08)$. There was no apparent deleterious impact on patients caused by the change in practice. In fact, mortality decreased in the postintervention period, although the before-after design of this study and a significant difference in baseline APACHE (Acute Physiology and Chronic Health Evaluation) scores between the preintervention and postintervention populations preclude confidence in this finding.

The demonstration by Conway Morris et al that exclusive use of BAL might lower a hospital's VAP rate by up to three-quarters will be very enticing to administrators striving to project their hospital in the best light possible. The study begs the question, however, of what constitutes truth in VAP surveillance. Is BAL-based surveillance a more accurate reflection of patients' morbidity or is it merely gaming the VAP definition to make one's VAP rates look as low as possible without meaningfully improving patient care?

In truth, there is no universally accepted reference standard for diagnosis of VAP. Conway Morris and colleagues implicitly endorse BAL by using it as the comparison standard to evaluate endotracheal aspirates. In addition, they used their findings to marshal a practice improvement initiative dedicated to increasing the use of BAL. There is no reason, however, to believe that $\mathrm{BAL}$ diagnosis is any closer to the truth than endotracheal aspirates. Studies assessing the sensitivity and specificity of BAL versus endotracheal aspirates relative to lung histology show little consistent difference. The reported sensitivity of BAL ranges from $11 \%$ to $77 \%$ while specificity ranges from $42 \%$ to $94 \% .^{2-6}$ Likewise, the sensitivity of endotracheal aspirate is $56-69 \%$ while the specificity is $75-95 \% .^{3-5}$ As such, both techniques miss many cases of VAP and inappropriately label some people as having the disease.

BAL's lack of discriminating power is borne out in Conway Morris and colleagues' own data. They found that patients suspected of having VAP had identical mortality rates regardless of whether their BAL culture was positive or negative. Likewise, their data on antibiotic usage indicates that clinicians take BAL results with a pinch of salt: they found that clinicians stopped antibiotics within 1.5 days following a negative endotrachael aspirate but continued treating patients for a mean of 5.8 days following a negative BAL culture.

One might be tempted to recommend that VAP benchmarking proceed albeit with a modified definition that explicitly stipulates quantitative BAL as the only diagnostic standard. This is unrealistic and dangerous. It is unrealistic because some patients are not candidates for bronchoscopy (typically those that require high levels of ventilator support). It is dangerous because bronchoscopy is an invasive procedure with small but real risks including pneumothorax, bleeding and oxygen desaturation.

A safer alternative might be to establish a weighting system to adjust a hospital's VAP rate according to the diagnostic method used. VAPs diagnosed using endotracheal aspirate cultures, which tend to be positive more often than BAL, should count less towards an institution's overall VAP rate than VAPs diagnosed using bronchoscopy. This method would be fairer than the current system, but is still flawed to the extent that it fails to take into account the interobserver variability and lack of specificity in the rest of the VAP definition. ${ }^{7}$ Patients are selected for microbiological sampling on the basis of clinical signs such as fever, abnormal leucocyte count, purulent sputum and radiographic infiltrates. These signs are notoriously non-specific and subject to substantial differences in interpretation between observers. ${ }^{8}$ Limiting the diagnosis of VAP to patients with positive BAL cultures or adjusting VAP rates according to sampling method will not obviate variability in selecting patients for investigation in the first place.

Conway Morris and colleagues argue that increased uptake of BAL is better care because it will lower antibiotic usage, which in turn will lower costs, limit antibiotic adverse effects and decrease selection for antibiotic-resistant organisms. In fairness to them, their study was begun at a time when best available evidence did suggest that preferential use of BAL could not only lower antibiotic usage but also decrease mortality. ${ }^{9}$ More recent studies, however, have not confirmed this finding. ${ }^{10}$ A large trial assessing clinical outcomes amongst patients with suspected VAP who were randomised to BAL with quantitative cultures versus endotracheal aspirates with non-quantitative cultures found no difference in patient mortality, length of stay in the ICU or hospital, or antibiotic usage. ${ }^{11}$ The trend towards lower antibiotic use and decreased mortality in the study of Conway Morris et al does not trump this randomised controlled trial given the before-after study design and lower APACHE scores in the postintervention group.

It is an irony of the quality improvement movement that the drive for zero hospital-acquired infections might occasionally do patients more harm than good. In the case of VAP, the looseness of the surveillance definition makes this a particular concern. Hospitals might be tempted to encourage an expensive and invasive diagnostic technique that has been largely discredited for routine practice by clinical trial data. This brings out a disturbing tension between hospital surveillance and clinical care in the case of VAP. The purpose of both surveillance and clinical care is to improve outcomes for patients. Subjectivity and inaccuracy in the VAP definition, however, allow 
room for hospitals to undertake practices that will markedly decrease their VAP rates and yet do little or nothing to improve patient outcomes. ${ }^{12}$

The implications are as follows: (1) VAP as currently defined is not a suitable measure for benchmarking hospitals or for external assessment of quality of care since the diagnosis is too inexact and liable to be gamed; (2) internal quality improvement initiatives to decrease VAP rates need to pay close attention to the diagnostic protocol to ensure consistency over time; and (3) we need a new, more objective and accurate measure for quality of care in ventilated patients that will reliably predict patients' outcomes. Until then, quality improvement initiatives would do well to track directly patient outcomes, such as duration of ventilation, length of stay and mortality, to increase confidence that an observed change in VAP rates translates into improved patient outcomes. This is critical to avoid unintended consequences from well-meaning initiatives to drive our hospital-acquired infection rates to zero.

Acknowledgements: The author wishes to thank Richard Platt MD, MSc for thoughtful comments on an earlier version of this manuscript.

Competing interests: None.

Thorax 2009;64:463-465. doi:10.1136/thx.2009.114165

\section{REFERENCES}

1. Conway Morris A, Kefala K, Simpson AJ, et al. Evaluation of the effect diagnostic methodology on the reported incidence of ventilator-associated pneumonia. Thorax 2009;64:516-22.

2. Kirtland $\mathbf{S H}$, Corley DE, Winterbauer $\mathrm{RH}$, et al. The diagnosis of ventilator-associated pneumonia: a comparison of histologic, microbiologic, and clinical criteria. Chest 1997;112:445-57.

3. Papazian L, Thomas P, Garbe L, et al. Bronchoscopic or blind sampling techniques for the diagnosis of ventilator-associated pneumonia. Am J Respir Crit Care Med 1995;152:1982-91

4. Fabregas N, Ewig S, Torres A, et al. Clinical diagnosis of ventilator associated pneumonia revisited: comparative validation using immediate post-mortem lung biopsies. Thorax 1999;54:867-73.

5. Marquette $\mathbf{C H}$, Copin MC, Wallet F, et al. Diagnostic tests for pneumonia in ventilated patients: prospective evaluation of diagnostic accuracy using histology as a diagnostic gold standard. Am J Respir Crit Care Med 1995;151:1878-88

6. Torres A, el-Ebiary M, Padro L, et al. Validation of different techniques for the diagnosis of ventilatorassociated pneumonia. Comparison with immediate postmortem pulmonary biopsy. Am J Respir Crit Care Med 1994;149:324-31.

7. Klompas M, Kulldorff M, Platt R. Risk of misleading ventilator-associated pneumonia rates with use of standard clinical and microbiological criteria. Clin Infect Dis 2008:46:1443-6.

8. Schurink CA, Van Nieuwenhoven CA, Jacobs JA, et al. Clinical pulmonary infection score for ventilator-associated pneumonia: accuracy and inter-observer variability. Intensive Care Med 2004:30:217-24.

9. Fagon JY, Chastre J, Wolff M, et al. Invasive and noninvasive strategies for management of suspected ventilator-associated pneumonia. A randomized trial. Ann Intern Med 2000;132:621-30.

10. Shorr AF, Sherner JH, Jackson WL, et al. Invasive approaches to the diagnosis of ventilator-associated pneumonia: a meta-analysis. Crit Care Med 2005:33:46-53

11. Heyland D, Dodek P, Muscedere J, et al. A randomized trial of diagnostic techniques for ventilator-associated pneumonia. N Engl J Med 2006;355:2619-30.

12. Klompas M, Platt R. Ventilator-associated pneumonia - the wrong quality measure for benchmarking. Ann Intern Med 2007;147:803-5.

\section{The fruits of our efforts: time for a different view of lung cancer and CT screening}

\section{Frank Detterbeck}

Lung cancer continues to be by far the leading cause of cancer deaths, primarily because it is usually not found until it is in a relatively advanced stage. As a result, a great deal of effort has focused on using CT imaging to screen a broad population. The two most influential papers on CT screening for lung cancer published recently have drawn conclusions that are diametrically opposed. ${ }^{12}$ Henschke et al ${ }^{1}$ found compelling evidence that CT screening would save many patients from death, while Bach et al concluded that CT screening may lead to harm through overtreatment of inconsequential lung cancers. It is worth taking a closer look at how two thoughtful groups can arrive at such disparate views.

The paper by Henschke et a $l^{1}$ reported a 5 -year survival rate of $86 \%$ for patients with lung cancer detected by CT screening. This is dramatically better than the

Correspondence to: Dr F C Detterbeck, Yale University, New Haven, MA 06520-8062, USA; frank.detterbeck@ yale.edu
5 -year survival rate of $16 \%$ for patients with lung cancer detected by routine care and reported through the US national cancer database. ${ }^{3}$ The implication is that CT screening has changed the outcome of the patients by early detection and early initiation of treatment.

The paper by Bach et al compared the results of three single-arm CT screening studies with predicted results using a model derived from and validated in patients with lung cancer detected during routine care as it exists currently. The number of lung cancers and lung cancer resections that actually occurred in the CT screened cohort was much higher than what was predicted by the model, whereas the number of deaths from lung cancer matched closely. The authors therefore concluded that CT screening resulted in overdiagnosis and overtreatment of indolent lung cancers that are of no clinical consequence in a substantial proportion of patients without any benefit. ${ }^{2}$

Some of the discussion of these papers centred around funding sources and whether there was potential for a bias as a result. ${ }^{4}$ The real issue, however, is to understand why the data from these two studies seem so dissimilar.

An emerging concept is that lung cancer involves a spectrum of tumours ranging from some that are very aggressive to others that are very indolent..$^{5-10}$ Importantly, it is becoming clear that CT screening in particular selects a population of patients that has a different spectrum of disease than that of patients detected by routine care. For example, a systematic review showed that the average volume doubling time of lung cancers detected during routine care was approximately 135 days compared with almost 500 days for lung cancers detected by CT screening. ${ }^{5}$ The proportion of patients with long doubling times (>400 days) increased from $3 \%$ in patients detected by routine care to $27 \%$ in those detected by CT screening. ${ }^{5-10}$ Thus, a comparison of lung cancers detected by CT screening with those detected by routine care is like comparing apples with oranges.

The comparison inherent in the paper by Henschke et al ${ }^{1}$ between lung cancers detected by CT screening and those detected during routine care is therefore flawed if the spectrum of disease is different in these two cohorts. Metaphorically, it is inappropriate to conclude from the observed differences that CT screening converted apples into oranges. Instead, one should conclude 